\title{
Hydroxychloroquine Myocardial Toxicity in a Patient with Systemic Lupus Erythematosus
}

\author{
Pierre Malchair $^{1}$, Maria Labori ${ }^{1}$, Manuel Rubio-Rivas ${ }^{1}$, Joel Salazar-Mendiguchia ${ }^{2}$, Nuria Baixeras ${ }^{3}$, Xavier Corbella ${ }^{1,4}$ \\ ${ }^{1}$ Department of Internal Medicine, Bellvitge University Hospital - IDIBELL, L'Hospitalet de Llobregat, Barcelona, Spain \\ ${ }^{2}$ Cardiomyopathy, Heart Failure and Transplantation Unit, Department of Cardiology, Bellvitge University Hospital - IDIBELL, L'Hospitalet de Llobregat, \\ Barcelona, Spain \\ ${ }^{3}$ Department of Pathology, Bellvitge University Hospital - IDIBELL, L'Hospitalet de Llobregat, Barcelona, Spain \\ ${ }^{4}$ Faculty of Medicine and Health Sciences, Universitat Internacional de Catalunya, Barcelona, Spain
}

Received: 24/03/2015

Accepted: 25/03/2015

Published: 08/05/2015

How to cite this article: Malchair P, Labori M, Rubio-Rivas M, Salazar-Mendiguchia J, Baixeras N, Corbella X. Hydroxychloroquine myocardial toxicity in a patient with systemic lupus erythematosus. EJCRIM 2015;2:doi: 10.12890/2015_000204

Conflicts of Interests: The authors declare that they have no conflicts of interest in this research

This article is licensed under a Commons Attribution Non-Commercial 4.0 License

\begin{abstract}
Hydroxychloroquine is an antimalarial drug used in many rheumatologic and systemic diseases. Although considered by clinicians to be relatively safe, serious side effects have been documented (retinotoxicity, neuromyotoxicity and cardiotoxicity).

We present the case of a 41-year-old woman with systemic lupus erythematosus (SLE) who presented at our institution with acute heart failure after taking hydroxychloroquine for a period of 3 months. An endomyocardial biopsy ruled out myocarditis related to systemic lupus erythematosus but demonstrated pathological changes related to hydroxychloroquine toxicity. It is exceptional to observe such cardiac toxicity after such a low cumulative dose (16 grams). The potential severity and reversibility of this complication underscores the importance of a high level of suspicion and timely diagnosis.
\end{abstract}

\section{LEARNING POINTS}

- Since the use of hydroxychloroquine in systemic lupus erythematosus (SLE) patients is increasing, clinicians should be alert to the development of antimalarial cardiotoxicity during treatment.

- In general, hydroxychloroquine myocardial toxicity should be suspected in all patients with cardiac complaints; new onset of heart failure or conduction abnormalities who received high cumulative dose; however, exceptionally, it can also be observed in SLE patients with lower doses after shorter periods of time.

- The potential reversibility of cardiotoxicity after antimalarial drug suppression is possible thanks to a rapid and accurate diagnosis, which is important for assuring appropriate management of the condition.

\section{KEYWORDS}

Hydorxychloroquine, cardiotoxicity, heart failure, side effect, systemic lupus erythematosus

\section{INTRODUCTION}

Hydroxychloroquine and chloroquine are antimalarial drugs extensively used in the long-term treatment of many rheumatologic and systemic diseases ${ }^{[1]}$. The most common side effects reported have been nausea, stomach cramps and diarrhea. After prolonged treatment, other side effects can appear. Cardiac toxicity due to hydroxychloroquine is a very rare complication, with cardiac conduction abnormalities the most reported adverse effects associated with this complication. Serious life-threatening infiltrative cardiomyopathy has also been reported in very few cases; thus, to date, the host factors that contribute to cardiotoxicity remain unclear. Some authors have suggested that a high cumulative dose could be the main risk factor of such toxicity ${ }^{[2,3]}$.

\section{CASE REPORT}

A 41-year-old woman with systemic lupus erythematosus (SLE) was admitted to the hospital because of one month-history of shortness of breath, orthopnea and legs edema. Eight years prior to admission, the patient was diagnosed with autoimmune hepatitis and primitive biliary cirrhosis (overlap syndrome), receiving ursodesoxicolic acid (700mg/d) with satisfactory clinical and analytical response. One year prior to admission, the patient was diagnosed with SLE after presenting legs and eyelids edema, recurrent joint pain and skin lesions on sunexposed areas. A renal biopsy revealed type $\mathrm{V}$ lupus glomerulonephritis. In accordance, the patient began taking oral prednisone (50mg/d) with an apparent clinical improvement but the persistence of proteinuria $(>7 \mathrm{~g} / \mathrm{d}$ ) lead her nephrologist to then prescribe mycophenolate 
followed by tacrolimus with no proteinuria improvement. Three months prior to admission, in addition to prednisone, mycophenolate and tacrolimus, the patient started hydroxychloroquine for the persistence of skin lesions.

After three months of hydroxicloroquine treatment, the patient was seen at the emergency department for a one-month gradual presentation of legs edema, orthopnea and paroxysmal nocturnal dyspnea. She had no prior history of lung or cardiovascular diseases. On admission, a physical examination, medical history check and chest X-ray confirmed signs of congestive heart failure without hemodynamic instability. The blood analysis was normal; hepatic and renal function indices were normal and the cardiac markers curve was negative. The electrocardiogram showed sinus rhythm with a right bundle branch block without ischemic signs.

With the suspicion of acute biventricular heart failure related to SLE, diuretics, vasodilatadors and high doses of prednisone were started, while hydroxychloroquine was stopped, in anticipation of further testing. During admission, laboratory tests revealed normal levels of Acute-phase proteins, elevated ProBNP $(8959 \mathrm{ng} / \mathrm{L})$ and low albumin $(26 \mathrm{~g} / \mathrm{L})$. All the antibodies and complement titres were within normal limits. A transthoracic echocardiography and cardiac magnetic resonance imaging (CMR) showed moderate left ventricular dilatation with eccentric hypertrophy, severe left ventricular systolic dysfunction (LVEF of 28\%) with global left ventricle (LV) hypokinesia and complete lateral akinesia. The right ventricle's function was normal. Myocardial edema was not found. Amyloidosis was ruled out by abdominal fat aspiration biopsy. Fifteen days after admission, a cardiac catheterization did not demonstrate coronary artery disease and an endomyocardial biopsy was performed. The biopsy specimen revealed the classic electron microscopic findings of myelin figures (myeloid bodies), which was highly suggestive of toxic myocardiopathy due to the hydroxychloroquine treatment; the biopsy findings, however, were not able to demonstrate the presence of curvilinear bodies.

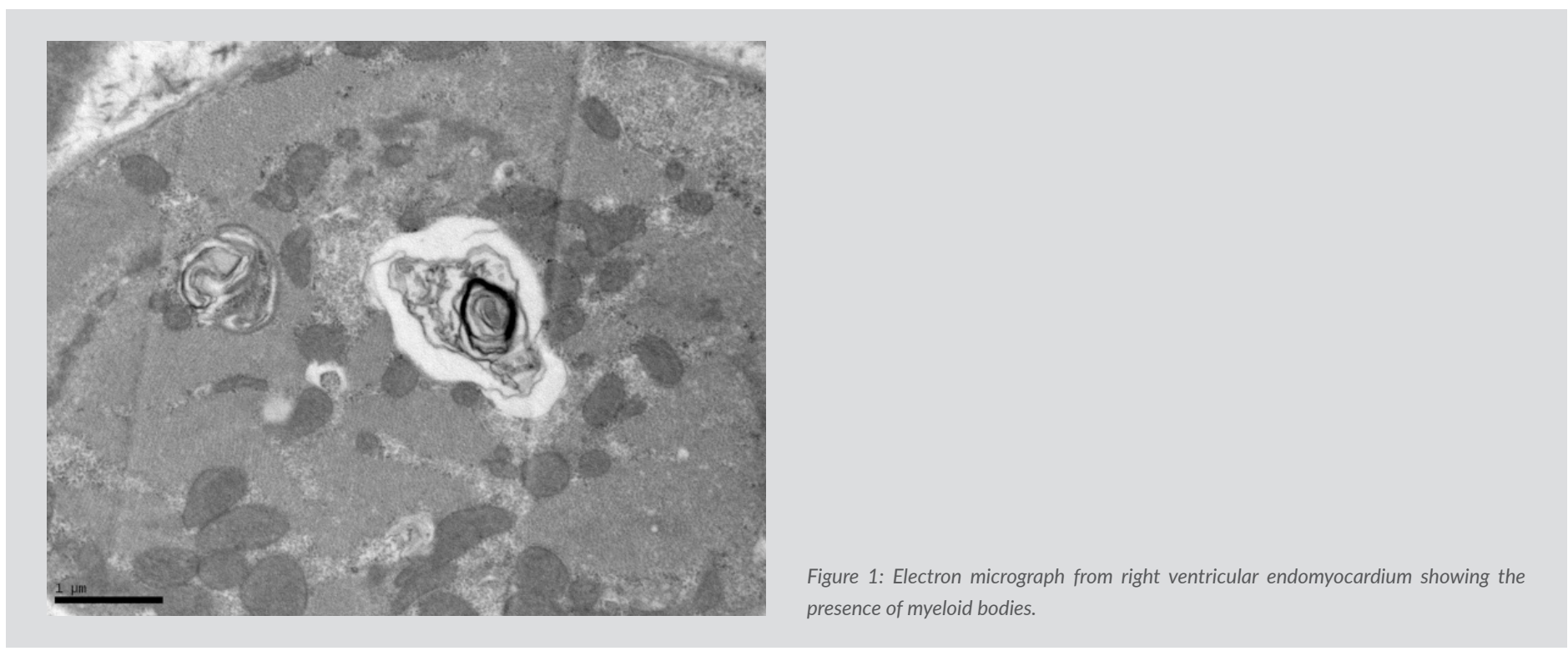

Three months after discharge, a new transthoracic echocardiography and CMR showed the persistence of LV dilatation without any improvement of neither LV systolic dysfunction (LVEF of 30\%), nor akinetic segments. Late gadolinium enhancement studies of both CMR support a diagnosis of nonischemic disease, while the absence of myocardial edema reasonably excludes myocarditis.

\section{DISCUSSION}

Cardiac involvement is one of the main complications contributing to the morbidity and mortality of patients suffering from SLE ${ }^{[4]}$. Involvement of any of the three layers of the heart - pericardium, myocardium and endocardium - have been demonstrated in more than $50 \%$ of SLE patients, when very sensitive techniques for cardiovascular investigation were used ${ }^{[4]}$. When myocardial is affected, myocarditis is the most characteristic clinical feature, presenting in a range of 3\% to $15 \%$ of SLE patients, though it appears to be much more common in autopsy studies, thus suggesting a subclinical nature of this complication.

Cardiotoxicity caused by some drugs used for lupus treatment, especially antimalarial drugs (chloroquine/hydroxychloroquine), is among the other causes of serious cardiac affectation in SLE patients. Host factors that contribute to cardiotoxicity caused by antimalarial drugs still remain unclear, but it is well known that large cumulative doses raise the likelihood for toxic myocarditis. Two different forms of cardiac toxicity have been described in relation to antimalarial drugs, with the most frequently reported being those related to conduction abnormalities, such as incomplete or complete right and left bundle-branch block or atrioventricular block ${ }^{[4]}$. Much less frequently, infiltrative cardiomyopathy has also been occasionally described as a serious complication.

Definitive diagnosis is provided by endomyocardial biopsy, revealing preserved myocardial architecture and a constellation of findings, including vacuolar myopathy, myeloid bodies and curvilinear bodies, which are considered the most specific findings when present. In spite of hydroxychloroquine withdrawal, the patient prognosis remains uncertain among the overall cases reported, varying from complete recovery to the need for cardiac transplantation, or death ${ }^{[5]}$.

In our case, optic and electron microscopy ruled out SLE-related inflammatory cardiomyopathy and other storage cardiomyopathy. Also, CMR results excluded ischemic cardiomyopathy. Although curvilinear bodies could not be found in our patient's endomyocardial biopsy, 
the diagnosis was supported by the lack of inflammatory activity and the presence of granular inclusions as myeloid bodies on the electron microscopy. Interestingly, the particularity of our patient was the relative rapid onset of hydroxichloroquine cardiomyotoxicity after a 3-month period of treatment and, subsequently, a relative low cumulative dose of hydroxychloroquine (16 grams). Our literature review showed only one previously reported similar case, involving a patient with recurrent malaria, who was treated repeatedly with chloroquine and presented myocardiotoxicity, with a similar low cumulative dosage of 15 grams $^{[6]}$.

\section{REFERENCES}

1. Ruiz-Irastorza G, Ramos-Casals M, Brito-Zeron P, Khamashta MA: Clinical efficacy and side effects of antimalarials in systemic lupus erythematosus: a systematic review. Ann Rheum Dis 2010; 69:20-28. 2. Ochhsendorf FR, Runne U: Chloroquine and hydroxychloroquine: side effect profile of important therapeutic drugs. Hautarzt 1991; 42:140-6.

3. Tönnesmann E, Stroehmann I, Kandolf R, Wolburg H, Strach K, Musshoff F, Tiemann K, Lewalter T: Cardiomyopathy caused by longterm treatment with chloroquine: a rare disease, or a rare diagnosis? The Journal of rheumatology 2012; 39:1099-1103.

4. Costedoat-Chalumeau N, Hulot JS, Amoura Z, Leroux G, Lechat P. Funck-Brentano C, Piette JC: Heart conduction disorders related to antimalarials toxicity: an analysis of electrocardiograms in 85 patients treated with hydroxychloroquine for connective tissue diseases. Rheumatology 2007; 46:808-810.

5. Tönnesmann E, Kandolf R, Lewalter T: Chloroquine cardiomyopathy-a review of the literature. Immunopharmacology and immunotoxicology 2012; 35:434-442.

6. Fragasso G, Sanvito F, Baratto F, Martinenghi S, Doglioni C, Margonato A: Cardiotoxicity after low-dose chloroquine antimalarial therapy. Heart Vessels 2009; 24:385-387. 